\title{
Epstein-Barr Virus-Positive Adrenal Diffuse Large B-Cell Lymphoma after Treatment for Angioimmunoblastic T-Cell Lymphoma
}

\author{
Akiko Hashimoto $^{1}$, Satsuki Asai ${ }^{2}$, Yasuhiro Tanaka ${ }^{1}$, Isaku Shinzato ${ }^{1}$ \\ ${ }^{1}$ Department of Hematology and Clinical Immunology, Kobe City Nishi-Kobe Medical Center, Japan \\ ${ }^{2}$ Department of Pathology, Kobe City Nishi-Kobe Medical Center, Japan
}

Received: 30/03/2021

Accepted: $11 / 10 / 2021$

Published: $27 / 10 / 2021$

\begin{abstract}
How to cite this article: Hashimoto A, Asai S, Tanaka Y, Shinzato I. Epstein-Barr virus-positive adrenal diffuse large B-cell lymphoma after treatment for angioimmunoblastic T-cell lymphoma. EJCRIM 2021;8: doi:10.12890/2021_002533.
\end{abstract}

Conflicts of Interests: The authors declare there are no competing interests.

Acknowledgements: We would like to thank the medical staff at Nishi-Kobe Medical Center who were involved in the care of this patient.

This article is licensed under a Commons Attribution Non-Commercial 4.0 License

\section{ABSTRACT}

Angioimmunoblastic T-cell lymphoma (AITL) can be complicated by Epstein-Barr virus (EBV)-positive B-cell lymphoma. B-cell lymphoma may develop simultaneously at the time of AITL diagnosis or after treatment for AITL. EBV-associated B-cell lymphoma can occur in nodal and extranodal sites. We report a case of EBV-positive diffuse large B-cell lymphoma (DLBCL) of the left adrenal gland that developed after treatment for AITL.

The patient presented with systemic lymphadenopathy and biopsy of one lymph node showed AITL. A complete response (CR) was achieved after initial chemotherapy for AITL, but 9 months later the left adrenal gland was enlarged. The diagnosis of EBV-positive DLBCL was made based on the histopathological findings of the left adrenal gland biopsy. Thus, EBV-positive DLBCL developed after AITL CR was achieved. Multi-drug chemotherapy combined with rituximab was administered for adrenal DLBCL, but only a partial response was achieved.

We confirmed that EBV-positive B-cell lymphoma developed after treatment for AITL. An adrenal primary is rare, and this is only the second case of EBV-positive B-cell lymphoma to be reported after treatment for AITL. Clinicians should keep in mind that when nodal and extranodal lesions are seen after AITL treatment, another biopsy should be performed for the accurate determination of whether these lesions indicate AITL relapse or new-onset EBV-positive B-cell lymphoma.

\section{LEARNING POINTS}

- We report a case of EBV-positive B-cell lymphoma of the adrenal gland after treatment for angioimmunoblastic T-cell lymphoma (AITL)

- When patients present with signs and symptoms suggestive of relapse after AITL treatment, another biopsy should be performed for the accurate determination of whether these lesions indicate AITL relapse or new-onset of EBV-positive B-cell lymphoma.

- The involvement of extranodal sites may indicate a poor prognosis of EBV-positive B-cell lymphoma after AITL treatment.

\section{KEYWORDS}

Adrenal mass, diffuse large B-cell lymphoma, Epstein-Barr virus, angioimmunoblastic T-cell lymphoma 


\section{INTRODUCTION}

Angioimmunoblastic T-cell lymphoma (AITL) is one of the mature T/NK cell neoplasms characterized by generalized lymphadenopathy and immunological abnormalities. The course of AITL is variable, but overall, the prognosis is poor. Several authors have reported that B-cell lymphoma can develop during the clinical course of AITL and that Epstein-Barr virus (EBV) is associated with the development of B-cell lymphoma. Most cases of B-cell lymphoma that develop after AITL treatment involve nodal sites, but sporadic cases of extranodal sites have been reported. Here, we describe a case of EBV-positive adrenal B-cell lymphoma that developed after CR was achieved following treatment for AITL. This is the second reported case of adrenal B-cell lymphoma which developed after treatment for AITL.

\section{CASE DESCRIPTION}

A 70-year-old man was admitted to our hospital for the evaluation of generalized lymphadenopathy in November 2017. He had a history of cerebral infarction, myocardial infarction and diabetes mellitus. He attended hospital regularly.

Computed tomography (CT) incidentally showed multiple lymphadenopathies (Fig. 1a), although he did not have any symptoms. Positron emission tomography-computed tomography (PET-CT) showed the accumulation of fluorodeoxyglucose in multiple lymph nodes (bilateral cervical lymph nodes, submandibular lymph nodes, axillary lymph nodes, supraclavicular lymph nodes, mediastinum, para-aorta lymph nodes, bilateral inguinal lymph nodes, subcutaneous nodules in the chest and bilateral upper limbs, nasopharynx, tonsils) . Laboratory examination showed elevation of CRP (1.1 mg/dl; normal range 0-0.5 mg/dl), LDH (276 IU/I; normal range 120-230 IU/I) and sIL2R (8000 $\mathrm{U} / \mathrm{ml}$; normal range $121-613 \mathrm{U} / \mathrm{ml}$ ).

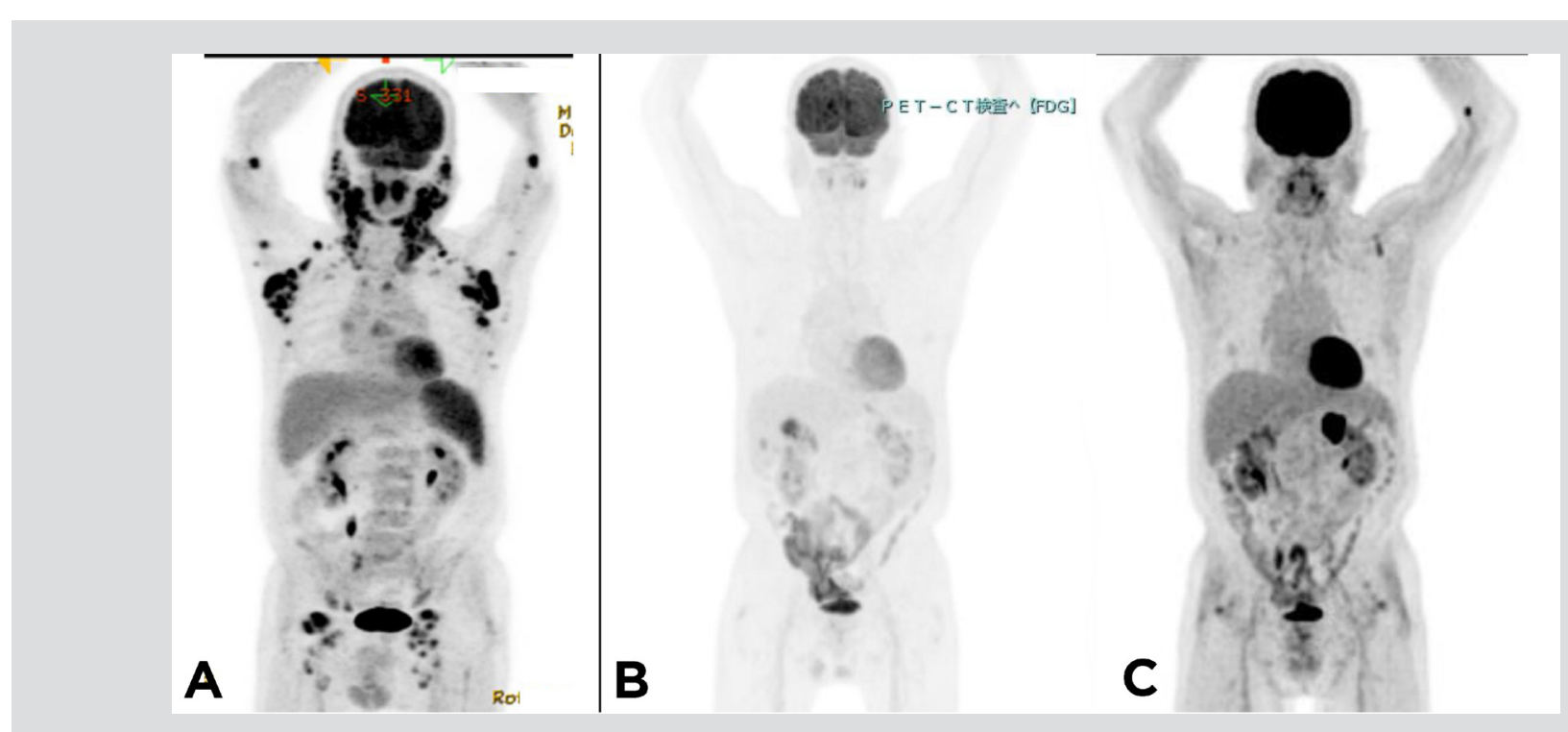

Figure 1. Sequential PET-CT scan images. (a) PET-CT images at angioimmunoblastic T-cell lymphoma (AITL) diagnosis showed abnormal accumulation of fluorodeoxyglucose in multiple lymph nodes. (b) PET-CT images after six cycles of CHOP chemotherapy for AITL showed no abnormal accumulations of fluorodeoxyglucose. (c) PET-CT images at diagnosis of EBVpositive adrenal diffuse large B-cell lymphoma showed abnormal accumulation of fluorodeoxyglucose only in the left adrenal gland

A left inguinal lymph node biopsy was performed in December 2017. Histopathological examination revealed small- to intermediate-sized lymphocytes and meshwork-like vascular proliferation. Immunohistochemical analysis showed that small to medium-sized abnormal lymphocytes were positive for CD3, CD4, CD5, BCL2, BCL6 and CCR4, and negative for CD8, CD10, CD56, CXCL13, TIA1 and ALK. Large abnormal cells expressed CD20 and CD30. In situ hybridization for the detection of Epstein-Barr virus-encoded small RNAs (EBERs) was positive only for large abnormal cells (Fig. 2). These results led to the diagnosis of angioimmunoblastic T-cell lymphoma (AITL) in accordance with the World Health Organization (WHO) 2016 classification. The RhoA G17V mutation was not detected in the biopsied lymph node. Tests for TET2, IDH2 and DNMT3A were not conducted. Chromosomal analysis using G-banding showed 46,XY, +1, der(1:15)(q10:q10) [7]/46,XY [13].Bone marrow examination was negative for lymphoma involvement. CHOP (cyclophosphamide $1370 \mathrm{mg} / \mathrm{m}^{2}$, doxorubicin 50 $\mathrm{mg} / \mathrm{m} 2$, vincristine $1.5 \mathrm{mg} / \mathrm{m}^{2}$, prednisolone $100 \mathrm{mg} / \mathrm{m}^{2}$ for 5 days) chemotherapy was administered every 3 weeks. After six cycles of CHOP chemotherapy, the patient achieved a complete metabolic response (CMR) as shown by a PET-CT scan in May 2018 (Fig. 1 b). 
Nine months later in April 2019, the patient complained of left-sided abdominal pain. CT detected a left adrenal mass. A PET-CT scan revealed accumulation of fluorodeoxyglucose only in the left adrenal mass (Fig. 1C). Laboratory examination showed many abnormalities including elevated CRP, LDH and sIL-2R. A CT-guided biopsy was performed.

Histopathological examination revealed large abnormal cells together with massive necrosis. Immunohistochemical analysis showed that large abnormal lymphocytes were positive for CD20 and CD30, and negative for CD3, CD5 and BCL6. In situ hybridization for the detection of EBERs was positive for large abnormal cells (Fig. 3). The serum EBV load was $9900 \mathrm{copies} / \mathrm{ml}$. These results led to the diagnosis of EBVpositive DLBCL, not otherwise specified (DLBCL-NOS) in accordance with the World Health Organization (WHO) 2016 classification. Thus, we considered that the patient had relapsed, with DLBCL-NOS presenting as a left adrenal mass after treatment for AITL. Chromosomal analysis using G-banding and JH rearrangement using Southern blotting was not carried out due to the very small volume of biopsied sample. After the diagnosis of DLBCL-NOS, the patient received three cycles of rituximab-THP-COP (THP (doxorubicin), cyclophosphamide, vindesine, prednisolone). However, due to insufficient efficacy, he received three cycles of rituximab-ICE (ifosfamide, carboplatine, etoposide) and achieved a partial response (PR). Nevertheless, his renal function deteriorated, and he died of renal failure in December 2019. An autopsy was not performed as permission was not obtained from his family
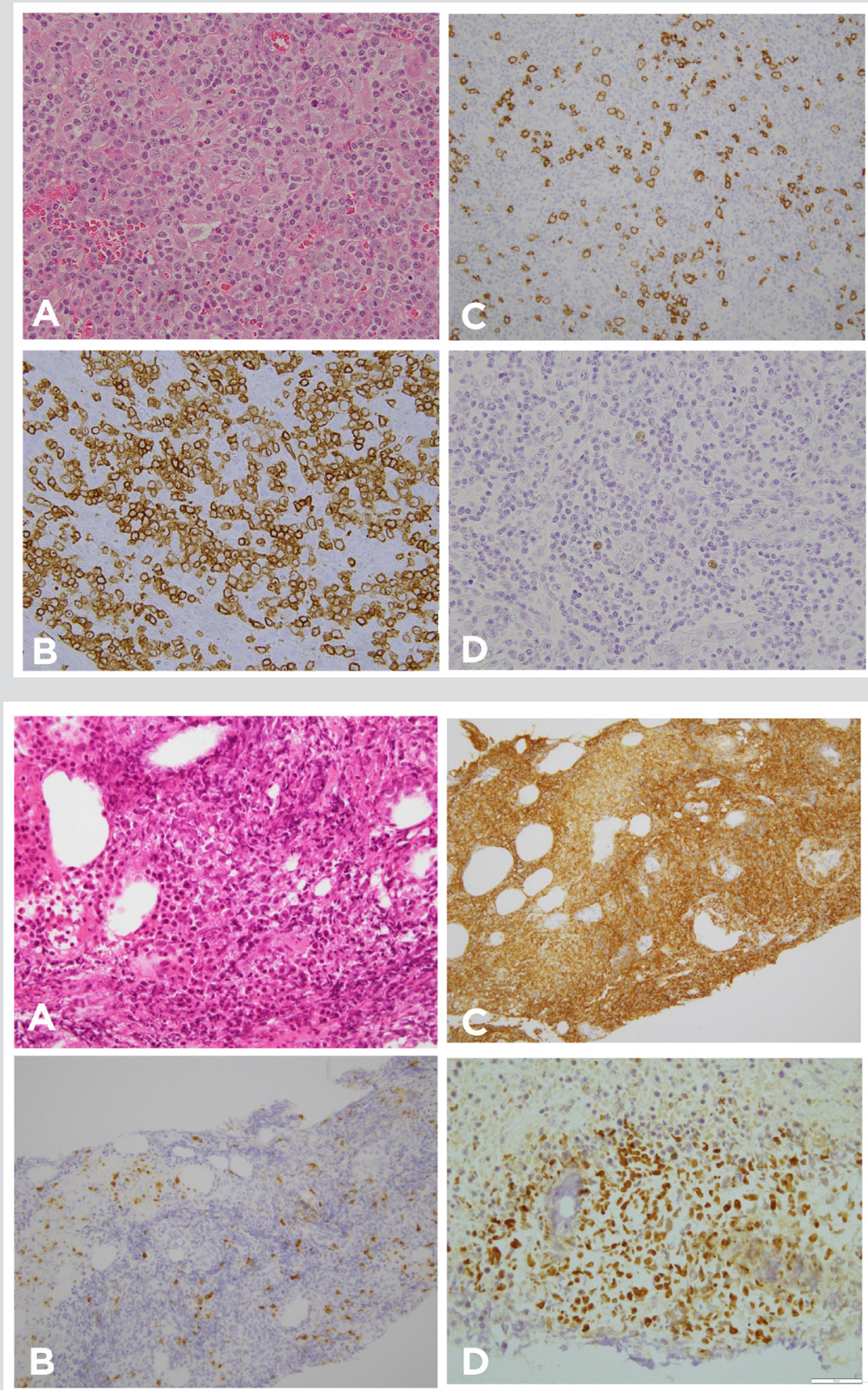
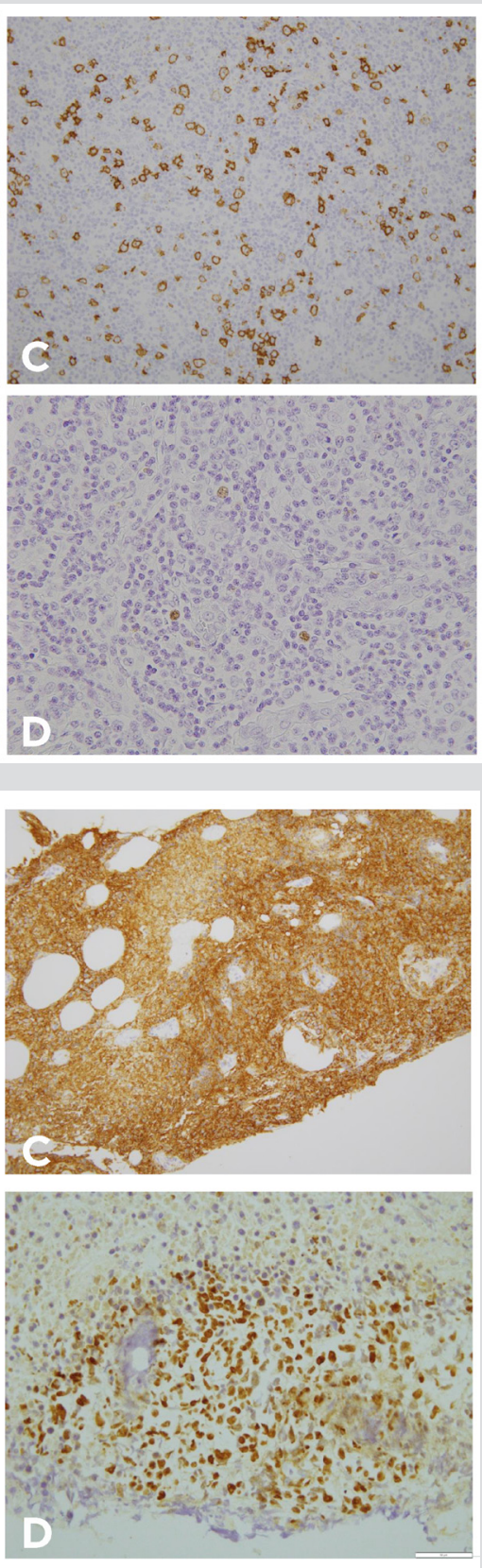

Figure 2. Histopathological findings of biopsied samples at angioimmunoblastic T-cell lymphoma (AITL) diagnosis. (a) Variously sized abnormal lymphocytes were detected in the biopsied lymph node, along with meshwork-like vascular proliferation (haematoxylin-eosin staining, original magnification, $\times 400$ ). (b) Immunohistochemical analysis showed that small to medium-sized abnormal cells were positive for CD3 (original magnification, $\times 400$ ). (c) Immunohistochemical analysis showed that large abnormal cells were positive for CD20 (original magnification, $\times 400$ ). (d) In situ hybridization to detect Epstein-Barr virus-encoded small RNAs (EBERs) was negative for small to medium-sized abnormal cells (original magnification, $\times 400$ )

Figure 3. Histopathological findings of biopsied samples following diagnosis of EBV-positive adrenal diffuse large B-cell lymphoma. (a) Large abnormal cells were found around the massive necrosis in the biopsied left adrenal tissue (haematoxylin-eosin staining, original magnification, $\times 400$ ). (b) Immunohistochemical analysis showed that large abnormal cells were negative for $\mathrm{CD} 3$ (original magnification, $\times 400$ ). (c) Immunohistochemical analysis showed that large abnormal cells were positive for CD20 (original magnification, $\times 400$ ). (d) In situ hybridization to detect Epstein-Barr virusencoded small RNAs (EBERs) was positive for large abnormal cells (original magnification, $\times 400$ ) 


\section{DISCUSSION}

Here, we report a rare case of EBV-positive left adrenal DLBCL after treatment for angioimmunoblastic T-cell lymphoma (AITL).

AITL had the characteristic local immune dysfunction in the tumour microenvironment, and EBV reactivation in $B$ cells could occur in local circumstances. This could lead to the proliferation of EBV-infected B cells and clonal expansion, and the development of B-cell lymphoma after AITL. Attygalle et al. reported that five cases of B-cell lymphoma developed during the clinical course of 31 cases of AITL ${ }^{[1]}$, and DLBCL was the most frequent histopathological type of B-cell lymphoma following AITL.

Most cases of DLBCL following AITL involved the lymph nodes, but a rare case of DLBCL developed as an extranodal lesion. Zhou et al. described 22 cases of EBV-positive DLBCL developing after an initial diagnosis of AITL ${ }^{[2]}$. Extranodal sites among these cases included the skin (three), and the bone marrow, lung, ileum, soft tissue and cerebellum (one each). Thus, as with our case, it is very rare for DLBCL to present as an adrenal mass during the clinical course of AITL.

To the best of our knowledge, only one case has been previously reported. Smeltzer et al. described a 59-year-old woman who was diagnosed with AITL presenting as skin nodules and palpable lymphadenopathy ${ }^{[3]}$. She was receiving vorinostat treatment for relapsed AITL. Both adrenal glands were enlarged. CT-guided biopsy was diagnostic for EBV-positive large B-cell lymphoma. This patient achieved complete remission after discontinuation of vorinostat followed by rituximab monotherapy. In our case, rituximab combination chemotherapy achieved PR, but our patient eventually died of renal failure, which was complicated by the chemotherapy.

Except for one patient with bone marrow involvement and one with cutaneous involvement, all reported patients with EBV-positive B-cell lymphoma in extranodal sites following AITL died due to infection or B-cell lymphoma progression, even though the B-cell lymphoma was localized in extranodal sites. Rituximab combination chemotherapy was not adopted for most cases of EBV-positive DLBCL due to the poor condition of the patients. This indicates that extranodal EBV-positive DLBCL may have a higher fatality rate than nodal disease following AITL albeit in localized extranodal sites. Further cases have clarified this point.

In conclusion, this is the second reported case of EBV-positive adrenal DLBCL-NOS after treatment for AITL. Clinicians should consider performing another biopsy of new lesions to distinguish between AITL relapse and new-onset EBV-positive B-cell lymphoma, because AITL is occasionally complicated with EBV-positive B-cell lymphoma and treatment with rituximab combination chemotherapy could improve the outcome. Nevertheless, involvement of extranodal sites in EBV-positive B-cell lymphoma following AITL may result in a poor prognosis even though the disease is only detected in a single lesion.

\section{REFERENCES}

1. Attygalle AD, Kyriakou C, Dupuis J, Grogg KL, Diss TC, Wotherspoon AC, et al. Histologic evolution of angioimmunoblastic T cell lymphoma in consecutive biopsies: clinical correlation and insights into natural history and disease progression. Am J Surg Pathol 2007;31:1077-1088.

2. Zhou Y, Rosenblum MK, Dogan A, Hungluth AA, Chiu A. Cerebellar EBV-associated diffuse large B cell lymphoma following angioimmunoblastic T cell lymphoma. J Hematopathol 2015;8:235-241.

3. Smeltzer JP, Viswanatha DS, Habermann TM, Patnaik MM. Secondary Epstein-Barr virus associated lymphoproliferative disorder developing in a patient with angioimmunoblastic T cell lymphoma on vorinostat. Am J Hematol 2012;87:927-928. 